\title{
"Like holding an umbrella before it rains": Acceptability of future rectal microbicides among men who have sex with men in India-A modified technology acceptance model
}

Venkatesan Chakrapani, Peter A. Newman, Murali Shunmugam, Shruta Mengle, Ruban Nelson, Clara Rubincam \& Pushpesh Kumar

Version Post-Print/ Accepted Manuscript

Citation Chakrapani, V., et al. (2017). "Like holding an umbrella before it rains": (published version) Acceptability of future rectal microbicides among men who have sex with men in India-A modified technology acceptance model. Qualitative Health Research 27(8): 1236-1248. doi: $10.1177 / 1049732317697947$. 
NOTE: This document is the authors' post-print (post-refereeing) version. The final publication is available at: http://journals.sagepub.com/doi/full/10.1177/1049732317697947

\section{Citation:}

Chakrapani, V., Newman, P. A., Shunmugam, M., Mengle, S., Nelson, R., Rubincam, C., \& Kumar, P. (2017). "Like holding an umbrella before it rains": Acceptability of future rectal microbicides among men who have sex with men in India-A modified technology acceptance model. Qualitative Health Research. [Published online ahead of print]. doi: 


\title{
"Like Holding an Umbrella Before It Rains": Acceptability of Future Rectal Microbicides Among Men Who Have Sex With Men in India-A Modified Technology Acceptance Model
}

Venkatesan Chakrapani ${ }^{1,2,3}$, Peter A. Newman ${ }^{4}$, Murali Shunmugam ${ }^{1}$, Shruta Mengle ${ }^{2}$, Ruban Nelson ${ }^{1}$, Clara Rubincam ${ }^{4}$, and Pushpesh Kumar $^{5}$

${ }^{1}$ Centre for Sexuality and Health Research and Policy, Chennai, India

${ }^{2}$ The Humsafar Trust, Mumbai, India

${ }^{3}$ PostGraduate Institute of Medical Education \& Research, Chandigarh, India

${ }^{4}$ University of Toronto, Toronto, Ontario, Canada

${ }^{5}$ University of Hyderabad, Hyderabad, India

\section{Corresponding Author:}

Peter A. Newman, Ph.D., Factor-Inwentash Faculty of Social Work, University of Toronto, 246 Bloor St. West, Toronto, Ontario, Canada M5S 1V4.; Email: p.newman@utoronto.ca

\begin{abstract}
Topical rectal microbicides (RMs) are a new prevention technology in development that aims to reduce the risk of HIV acquisition from anal sex. We examined RM acceptability among men who have sex with men (MSM) in India. We conducted a qualitative exploratory study guided by a modified Technology Acceptance Model, with 10 focus groups $(n=61)$ of MSM and 10 key informant interviews. Data were explored using framework analysis. RM acceptability was influenced by technological contexts: perceived usefulness of RMs, perceived ease of use of RM and applicator, and habits around condom and lubricant use; individual and interpersonal contexts: perceived relevance and preferences for product formulation and dosing frequency; and MSM community/social contexts: perceived social approval, RM-related stigma, social support. Implementation of RMs for MSM in India may be supported by multi-level interventions that engage community-based organizations in destigmatizing and distributing RMs, ideally gelbased products that enable on-demand use before sex.
\end{abstract}

\section{Keywords}

rectal microbicide; acceptability; HIVIAIDS; HIV prevention; men who have sex with men; male sex workers; focus groups; interviews; framework analysis; qualitative; India 


\section{Introduction}

India has the third largest HIV epidemic in the world, with an estimated 2.12 million people living with HIV (National AIDS Control Organization [NACO], 2016). Indian government data from select HIV surveillance sites indicate HIV prevalence among men who have sex with men (MSM) $(4.3 \%)$ is 16 times higher than that among the general population $(0.26 \%)$ and is increasing in several Indian cities (NACO, 2016). Recent data from a broader 12-city surveillance study document weighted HIV prevalence among MSM of $7.0 \%$ (range: $1.7 \%$ $13.1 \%$ ), with evidence suggesting escalating epidemics among MSM in some cities despite steady declines in HIV prevalence in the general population (Solomon et al., 2015). Despite rapid scale up of targeted interventions for MSM since 2006 by India's National AIDS Control Organization (2016), wide variation in condom use for anal sex (ranging from $58.1 \%$ to $97.0 \%$ ) has been identified among MSM in different states (Jha et al., 2014). In addition to promoting consistent condom use, it is vital to broaden options for HIV prevention among MSM in India.

Rectal microbicides (RMs) are topical antiretroviral-based products that are designed to be applied to the rectal mucosa to prevent or significantly reduce HIV acquisition during anal sex (McGowan, 2011). Initial research to develop vaginal microbicides was motivated by the need to create HIV prevention products under women's control, given escalating HIV infections among women globally and the fact that the predominant tool, the male condom, is ultimately under the control of men (Stein, 1990). As a substantial proportion of MSM do not use condoms consistently, and amid sustained disparities in HIV infections among MSM globally (Baral et al., 2015)_-particularly in low- and middle-income countries (Baral, Sifakis, Cleghorn, \& Beyrer, 2007) —efforts to develop RMs similarly aim to broaden the HIV prevention tools available to MSM, as well as to women (McGowan, 2011). On a biological level, condomless receptive anal sex has the single highest per-act risk of HIV acquisition, 10 to 20 times that of condomless vaginal sex (Vittinghoff et al., 1999). On social and structural levels, condom use is often encumbered by substantial barriers, such as low educational attainment, poverty, sex work, sexual and HIV stigma, and criminalization of sex between men, which have been documented among MSM in India (Chakrapani, Newman, Shunmugam, McLuckie, \& Melwin, 2007; Chakrapani, Shunmugam, Newman, Kershaw, \& Dubrow, 2015). Thus, even a partially efficacious RM may exert a substantial impact as part of a combination HIV prevention strategy -integrated with other biomedical, behavioral, and social approaches-in reducing the HIV burden among MSM.

Several RM candidates have been successfully tested in Phase 1 (Anton et al., 2011; Hiruy et al., 2015; McGowan et al., 2013) and Phase 2 clinical trials (MTN-017) (Cranston et al., 
2017). As in the case of any new health technology, effectiveness is contingent on both a product's efficacy and its acceptability to end users (Newman, Roungprakhon, Tepjan, \& Yim, 2010). RM acceptability refers to potential users' judgments of the satisfactoriness of future RMs and willingness to use them (Newman, Cameron, Roungprakhon, Tepjan, \& Scarpa, 2016). RM acceptability studies conducted among MSM and transgender women in both developed and developing countries have explored the impact of product formulation (gels, lubricants, suppositories, douches/enemas), mode of application, frequency and volume of application, side effects, cost, and relationship type on RM acceptability (Carballo-Diéguez et al., 2008; Cranston et al., 2017; Newman et al., 2016; Newman, Roungprakhon, \& Tepjan, 2013; Pines et al., 2013; Tang et al., 2016). These studies indicate that MSM and transgender women, including those engaged in sex work, are interested in a safe and effective RM, and that RM acceptability may be influenced by a range of factors at individual, interpersonal, and social levels (Newman et al., 2013). Limited previous investigations that explored vaginal microbicide acceptability among Indian women and men reported lubricating properties of the product as a facilitator, and fragrance as well as male partner's disapproval as barriers to acceptability (Greene et al., 2010; Joglekar et al., 2010; Tolley et al., 2006). However, we are aware of no published studies of RM acceptability in India.

As RMs are a new HIV prevention technology, the Technology Acceptance Model (Davis, 1989) provides a useful framework to explore RM acceptability. Several modified versions of the Technology Acceptance Model, originally from the field of information technology, have been applied to explore acceptance of other health technologies among potential or actual users (Aggelidis \& Chatzoglou, 2009; Gagnon, Orruno, Asua, Abdeljelil, \& Emparanza, 2012; Orruno, Gagnon, Asua, \& Abdeljelil, 2011). We adapted a variant of the Technology Acceptance Model initially developed to assess teledermatology adoption-since it included social environmental factors as well as barriers to acceptability (Orruno et al., 2011)_ to serve as an analytical framework for RM acceptability. In this model, intention to use a new technology depends on the following: (a) technological context: perceived usefulness and ease of use, and habits; (b) individual context: compatibility (existing values, previous experiences and needs of potential adopters), and attitudes; and (c) organizational context: facilitators and subjective norms (perceived social approval and social norms). Our focal research question was the following:

Research Question: What factors influence the acceptability of future RMs among MSM in India? 


\section{Method}

We used a qualitative exploratory design given this is among the first studies of RM acceptability with MSM in India and our interest in exploring a wide variety of perspectives (Brink \& Wood, 1998). In the last quarter of 2014, we conducted 10 focus groups among a purposive sample of five subgroups of MSM who differ by sexual self-identifications and behavior. Although not fixed (essential) categories, and with a degree of fluidity between sexual identifications and behaviors, the subgroups comprised the following: kothis (a culturally indigenous term for MSM with "feminine" gender expression who primarily adopt a receptive role in anal sex); panthis (a term used for MSM with "masculine" gender expression who primarily adopt an insertive sexual role with kothis); double-deckers (those who adopt both insertive and receptive sexual roles); gay-identified; and bisexual-identified MSM (Chakrapani et al., 2007; Newman, Chakrapani, Cook, \& Kakinami, 2008). Participants were recruited through community-based organizations (CBOs) working with MSM, including male sex workers, in Chennai (est. population 9.8 million) (Social Welfare Association for Men and Sahodaran) and Mumbai (est. population 20.7 million) (Humsafar Trust), metropolitan areas with large populations of MSM with sustained high HIV prevalence (Solomon et al., 2015). We did not screen participants individually by HIV status; however, we conducted recruitment at meetings and events for HIV-negative/unknown status MSM. Eligibility criteria for focus group participants were age 18 years or above, self-identified as MSM, and able to provide informed consent. Trained research staff employed at local CBOs recruited potential focus group participants by word of mouth.

Ten key informant interviews were conducted with health care providers and MSM community leaders in Chennai and Mumbai. We purposively selected key informants with indepth knowledge of MSM communities to provide additional perspectives to that of focus group participants to enrich our understanding and interpretation of the data (Patton, 1999).

The study protocol was approved by the institutional review boards of the University of Toronto and the Humsafar Trust. All participants provided written informed consent.

\section{Data Collection}

In Mumbai, six MSM participated in each of five focus groups $(n=30)$, and in Chennai, five to seven MSM participated in each of five focus groups $(n=31)$. We used a semi-structured focus group topic guide to explore and assess awareness and acceptability of RMs among MSM. The following domains were explored: prior knowledge of RMs, willingness to use a RM, perceived 
barriers and facilitators to future RM uptake, RM preferences, access, and anticipated impact on condom use.

Focus group (70-90 minutes) and key informant interviews (30-45 minutes) were conducted in participants' native language (Tamil in Chennai; Marathi or Hindi in Mumbai) by trained Indian facilitators/interviewers who were native speakers of these local languages. A few key informant interviews were conducted in English. Each focus group participant received INR 300 (5.00 USD) as compensation for his time.

After assessing focus group participants' prior knowledge of RMs, the group facilitator provided a standard explanation of a RM (adapted from factsheets of the Microbicides Trials Networks and Global Campaign for Microbicides) (International Rectal Microbicide Advocates, 2010; Microbicide Trials Network, 2013) and using a pictorial card in participants' native language. The pictorial cards were developed by the research team in India in consultation with local community leaders familiar with the study populations. Participants were encouraged to ask questions, and misunderstandings were clarified. As the actual efficacy of RMs is not yet known, participants were informed that, if applied every time before anal sex, RMs may offer RM efficacy on results from a Phase 3 vaginal microbicide trial that indicated $54 \%$ efficacy among highly adherent participants (Karim et al., 2010).

\section{Data Analysis}

Focus groups and key informant interviews were digitally recorded, transcribed verbatim and translated into English. Accuracy of transcription was assessed by two bilingual research coordinators who randomly selected $20 \%$ of transcripts and compared them with their respective audio files. Similarly, accuracy of translation was checked by the same bilingual research coordinators who compared random segments of the translated text with the native language transcripts.

Focus group and interview data were explored using framework analysis (Pope, Ziebland, \& Mays, 2000; Ritchie \& Lewis, 2003). Framework analysis is similar to thematic analysis, except the analysis is more focused, based on the topic guides, in this case on factors that may influence RM acceptability. We developed an initial coding framework based on a priori codes derived from the topic guides and existing literature on RM acceptability. Next, we identified inductive/emergent codes and categories from the data, which we added to the framework and used in further coding of the data. Differences in coding were discussed among two data analysts and senior investigators, and resolved by consensus. The analysis focused on identifying factors that may hinder or facilitate RM acceptability. 
In addition to the use of multiple coders and assessment of the accuracy of transcription and translation, we used several strategies to support methodological rigor. Member checking (Lincoln \& Guba, 1985) was conducted by discussing findings and interpretations in meetings with peer research teams in local research sites. Data source triangulation between MSM participants and key informant health care providers and community leaders supported our understanding from different perspectives (Denzin, 1978; Patton, 1999). Methodological triangulation of focus groups and interviews along with data source triangulation across diverse MSM from two cities supported data saturation: no new themes arose from the final focus groups and interviews (Denzin, 1978; Fusch \& Ness, 2015). Finally, prolonged engagement (Creswell \& Miller, 2000) in the field on the part of both lead investigators, each with 15 years of research experience with MSM in India, further enhances the credibility of the findings.

\section{Results}

\section{Sociodemographic Characteristics}

Focus group participants' $\left(n=61 ; M_{\text {age }}=26\right.$ years $[S D=4.8]$ ) sociodemographic characteristics are presented in Table 1. More than half $(54.1 \%)$ completed high school, and one-third $(32.8 \%)$ completed a college degree. More than three-fourths $(77.0 \% ; n=47)$ were employed $(14.9 \%$ [n $=7$ ] of these as sex workers), $13.1 \%(n=8)$ were college students, and $9.8 \%(n=6)$ unemployed. In addition to those who identified as sex workers, 19 (31.1\%) participants engaged in occasional sex for money. The majority $(78.7 \% ; n=48)$ were single. Participants were roughly equally divided among kothi $(21.3 \%)$, panthi $(19.7 \%)$, double-decker $(18.0 \%)$, gay $(19.7 \%)$, and bisexual $(21.3 \%)$ sexual self-identifications. 
Table I. Sociodemographic Characteristics of Focus Group Participants $(N=61)$.

\begin{tabular}{|c|c|c|c|c|}
\hline Characteristics & $\begin{array}{l}\text { Overall Sample } \\
\qquad(N=61)\end{array}$ & $\begin{array}{l}\text { Chennai } \\
(n=31)\end{array}$ & $\begin{array}{l}\text { Mumbai } \\
(n=30)\end{array}$ & $p$ Value \\
\hline \multicolumn{5}{|l|}{ Age (years) } \\
\hline M & 26.1 & 26.9 & 25.2 & \multirow[t]{2}{*}{.17} \\
\hline$S D$ & 4.8 & 4.3 & 5.3 & \\
\hline \multicolumn{5}{|l|}{ Monthly income, $\mathrm{INR}^{2}$} \\
\hline M & 12,245 & 10,685 & 14,350 & \multirow[t]{2}{*}{.01} \\
\hline \multirow[t]{2}{*}{ SD } & 6,066 & 5,394 & 6,416 & \\
\hline & $n(\%)$ & $n(\%)$ & $n(\%)$ & \\
\hline \multicolumn{5}{|c|}{ Highest level of completed education } \\
\hline$<$ High school & $8(13.1)$ & $6(19.4)$ & $2(6.7)$ & \multirow[t]{3}{*}{.01} \\
\hline High school/higher secondary & $33(54.1)$ & II (35.5) & $22(73.3)$ & \\
\hline College degree & $20(32.8)$ & $14(45.2)$ & $6(20.0)$ & \\
\hline \multicolumn{5}{|l|}{ Employment } \\
\hline Employed & $47(77.0)$ & $27(87.0)$ & $20(66.6)$ & \multirow[t]{3}{*}{.06} \\
\hline Unemployed & $6(9.8)$ & $2(6.4)$ & $4(13.3)$ & \\
\hline Student & $8(13.1)$ & $2(6.4)$ & $6(20.0)$ & \\
\hline \multicolumn{5}{|l|}{ Primary identity } \\
\hline Kothi & $13(21.3)$ & $7(22.6)$ & $6(20.0)$ & \multirow[t]{5}{*}{.00} \\
\hline Panthi & $12(19.7)$ & $6(19.4)$ & $6(20.0)$ & \\
\hline Double-decker & $11(18.0)$ & $11(35.5)$ & & \\
\hline Gay & $12(19.7)$ & & $12(40.0)$ & \\
\hline Bisexual & $13(21.3)$ & $7(22.6)$ & $6(20.0)$ & \\
\hline \multicolumn{5}{|l|}{ Marital status } \\
\hline Single & $48(78.7)$ & $25(80.6)$ & $23(76.7)$ & \multirow[t]{2}{*}{.70} \\
\hline Married & $13(21.3)$ & $6(19.4)$ & $7(23.3)$ & \\
\hline \multicolumn{5}{|l|}{ Current living situation } \\
\hline Alone & $19(31.1)$ & $9(29.0)$ & $10(33.3)$ & \multirow[t]{3}{*}{.12} \\
\hline With parents or wife & $38(62.3)$ & $18(58.1)$ & $20(66.7)$ & \\
\hline With peers & $4(6.6)$ & 4 (12.9) & & \\
\hline \multicolumn{5}{|l|}{ Sex work involvement } \\
\hline No & $35(57.4)$ & $12(38.7)$ & $23(76.7)$ & \multirow[t]{2}{*}{.00} \\
\hline Yes & $26(42.6)$ & $19(61.3)$ & $7(23.3)$ & \\
\hline \multicolumn{5}{|l|}{ Consistent condom use, past month } \\
\hline Yes & $41(67.2)$ & $24(77.4)$ & $17(56.7)$ & \multirow[t]{2}{*}{.08} \\
\hline No & $20(32.8)$ & $7(22.6)$ & $13(43.3)$ & \\
\hline \multicolumn{5}{|c|}{ Ever used water-based lubricants, past year } \\
\hline Yes & $26(42.6)$ & $16(51.6)$ & $10(33.3)$ & \multirow[t]{2}{*}{.14} \\
\hline No & $35(57.4)$ & $15(48.4)$ & $20(66.7)$ & \\
\hline
\end{tabular}

Note. INR = Indian rupee. ${ }^{2} 60$ INR $\sim 1$ USD.

More than two thirds $(67.2 \%, n=41)$ of participants reported having heard about waterbased lubricants for anal sex; $42.6 \%(n=26)$ reported having ever used water-based lubricants for anal sex in the past year, with or without condoms. No focus group participants and only one key informant had heard of "rectal microbicides" (in English or local languages). 
We describe factors that influence RM acceptability, including representative quotations from participants, in technological, individual and interpersonal, and social/ community contexts in accordance with the modified Technology Acceptance Model (see Figure 1).

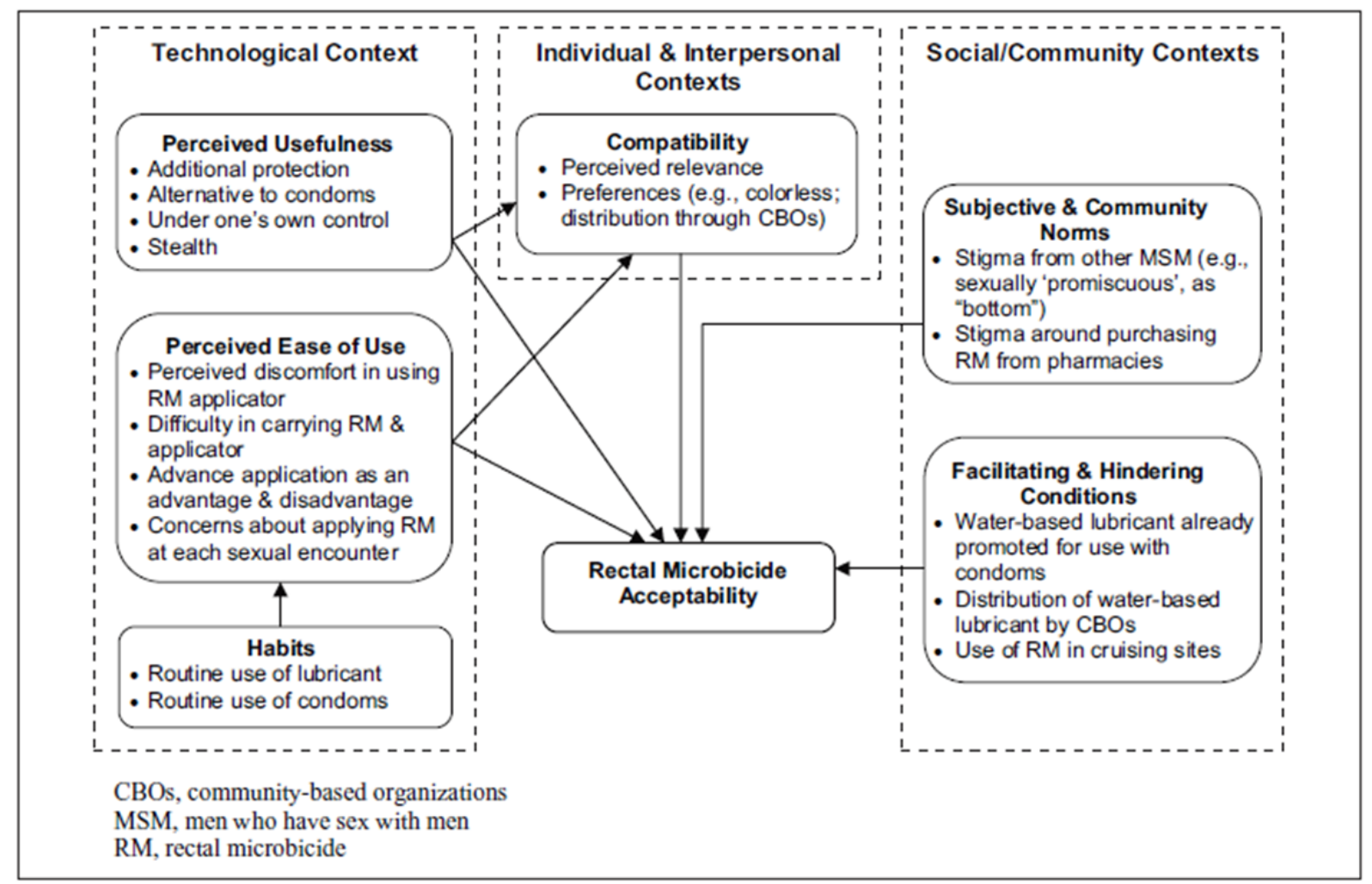

Figure I. Factors influencing acceptability of future rectal microbicides among men who have sex with men in India: A modified Technology Acceptance Model.

Note. $\mathrm{CBO}$ = community-based organizations; $M S M=$ men who have sex with $m e n ; R M=$ rectal microbicide.

\section{Technological Context}

Perceived usefulness. Participants reported that RMs would offer additional protection when used with condoms (in instances of condom breakage), could be used in the absence of condoms (in certain contexts such as when alcohol was consumed prior to sex), and may increase pleasure during anal sex. For example, participants said,

People might prefer using it because even if they get exposed to blood during anal sex the germ killer [RM] will kill the virus there itself. (Double-decker)

Both need to be used. Condom may offer 95 percent protection, but sometimes condom gets torn or damaged, at that situation, this thing [RM] which is inside the anal area will offer protection. (Panthi) 
Suppose he [casual partner] has HIV. We don't know and we are doing sex with condom and condom tears. Then his virus. . I I mean then his liquid will go inside our backside. Then the [RM] will work. (Gay man)

Some MSM noted not having to worry about the HIV status of their partner as a key advantage of RM use. Other perceived advantages were: RM use is under one's control, not determined by partners; stealth (ability to hide use or convince partners that they have applied an ordinary sexual lubricant). For instance, a double-decker said,

Some panthis [sexual partners] don't like using condoms. Since it can be applied a few hours before, I can apply it without their knowledge. And I can have sex with them without condoms. They will be satisfied and at the same time I will be also safe.

(Double-decker)

Divergent perspectives emerged as to how education and income-level might affect RM uptake. Some non-gay-identified MSM and MSM community leaders expressed that RMs may not be perceived as useful by higher socioeconomic MSM such as gay and bisexual-identified men, but may be perceived as useful by lower socioeconomic MSM in sex work. But a gayidentified man reported that educated MSM are more likely to use RMs: "Getting this gel [RM], doing this, doing that, will be done mostly by people who are educated and who have money" (gay man). Participants also expressed interest in knowing whether microbicides would work if one has multiple anal sex partners in a day or in group sex, without having to apply a RM prior to each sexual encounter.

Perceived ease of use. Use of RMs was perceived to pose several difficulties. The need to use a "syringe-like" applicator was viewed as a major disadvantage as it was seen as potentially painful, cumbersome, unhygienic, and impractical (if unplanned sex happens in cruising sites). For example, participants said,

Rather than applying through syringe it can be like water-based lubricants ... because it may not be possible to inject it every time after sex. (Kothi in sex work)

Who will take it [applicator] and push like this? ... One will prefer to apply directly from a tube. That is better. Who will keep it in hand carefully and carry? (Gay man) 
Community leaders stated that MSM from lower socioeconomic classes might find it difficult to use RMs:

I mean as far as condom is concerned you very certainly use one condom every time. That's all. In this [RM] product, one needs to know where to apply, how much to apply and how to apply properly. (MSM community leader)

Participants indicated concerns about where to store RMs and whether a family member might see them. A few participants were concerned that their regular male partners would suspect them of infidelity if they found out that RMs were being used without their knowledge. Concerns about side effects (e.g., risk of cancer from long-term use) were expressed by a few participants.

The need to apply microbicides a few hours before having anal sex was considered difficult by some participants, particularly as sex with casual partners may be unplanned. For instance, a double-decker commented that

If I am going for sex work then it is fine. It can be applied a few hours before. Consider I am going somewhere. I don't know whether I will come across a panthi and have sex with him. In such situation, applying it [RM] in advance is like holding an umbrella before it rains. (Double-decker)

The need to apply a RM every time before one has receptive anal sex was seen as a hassle for MSM in sex work and those who have group sex. As a gay-identified man pointed out, "Let us say I applied it 8 hours before. But for my second sex if I have to apply it then I have to find place to do so. It is a problem then" (gay man).

This view was shared by some community leaders as well:

[RM] may not suit those who have sex for money . . . they will have more encounters and re-applying each time after sex will not be possible . . . this gel will be used only if it offers protection for a longer time or else it will become a failure like female condoms. (MSM community leader)

Double-deckers shared their view that while they were willing to use a RM, it would be more difficult to convince their double-decker partners to adopt RM use:

We insert and also receive ... if we give 'gaans' [receptive anal sex] then we can apply it—not a problem. But if we ask them (partners) to apply it they will feel shy. They will think-“Why he is giving it to me?" (Double-decker) 


\section{Habit: Routine Use of Lubricant and Condoms}

Participants believed that MSM who access services from CBOs are more likely to use RMs if they are CBO-endorsed. This acceptance, they reasoned, may be supported by both improved health-seeking behavior of MSM who attend CBOs and the fact that they might have tried free water-based lubricants distributed by these CBOs.

MSM in sex work reported current use of water-based lubricants to enable painless sex and to prevent condom breakage, thus gaining protection against HIV infection. For instance, a participant said,

I go for sex work. The person who comes to me will not care about my protection. [But] I will use condom along with gel [water-based lubricant]. I know that it will not give full protection but it can prevent condom breakage. That's the reason for using it. (Double-decker)

Some participants, mostly panthis and gay men, believed that water-based lubricants were largely used by MSM with multiple partners or by MSM in sex work, and thus those groups are more likely to use RMs. For instance, a panthi said, "Those who do it for money would use this product. They will have more contacts. I mean 10 to 20 in a day" (panthi).

MSM participants and community leaders both indicated that if RMs become available, many MSM will stop using condoms with their regular male partners and with their casual partners, thus breaking the habit of condom use and promoting risk compensation. As a kothi reported,

MSM and transgender people would be happy. They would think that they can have condomless sex with known or 'cheese' [handsome] panthis. They will think "It is ok not to use condom now" and do it happily. (Kothi in sex work)

\section{Compatibility}

Compatibility entails the perceived fit between RM use and one's sexual roles and perceived risk.

Perceived relevance. Kothis reported that other kothis, especially those in sex work, would see RMs as relevant as they are primarily receptive partners, but panthis would not see RMs as relevant. Panthis tended to agree with this line of reasoning, as the responsibility for using any product related to anal sex was seen as that of their kothi (receptive) partners: 
Gel [RM] is only useful for those who receives (receptive partner). In the absence of condom, semen can go inside and can create problems. In such a situation gel will be useful for kothis. (Panthi)

Possible lubricating properties of RMs were seen as beneficial by kothis in sex work as they thought that use of RM will shorten the duration of sex with their paying partners. In contrast, RMs with lubricating properties were seen as a disadvantage by panthis, who generally eschew lubrication, as they felt it may reduce sexual pleasure and would be useful only for MSM who adopt a receptive role for the first time.

Although kothis reported that their regular panthi partners would not approve of their RM use, some panthis supported RM use by their kothi partners as they thought that a microbicide might protect the insertive partner as well: "If condom gets damaged during anal sex then this one [RM] inside his anus will protect me" (panthi). Similarly, a bisexual-identified man reported that he would allow his partner to use a RM as it is his responsibility to protect his partner's and his own health.

Class differences among subgroups of MSM seemed to influence their perceptions of compatibility in terms of which subgroups might use a RM, though sometimes revealing contradictions. For example, kothis reported that they would use a RM, but that educated gay men would not be interested as they feel invulnerable to HIV; however, gay-identified men indicated that they would use a RM, but believed that lower socioeconomic status MSM, such as kothis, would not use RMs because of the cost and difficulty in applying RMs with an applicator.

Preferences. Compatibility also entailed individual preferences for RM use. Participants generally preferred RMs that can serve as lubricants and, using existing water-based lubricants as a reference point, which are odorless and colorless_not red/pink or yellow, as the former resembles blood and the latter resembles fecal matter. Many preferred to apply RMs in a similar way to applying sexual lubricants-with no need for special applicators. One participant, however, expected a hygienic kit with applicator and RM tube so that the applicator and perianal area can be cleaned after use. Although participants acknowledged the practice of enema use among some MSM prior to anal sex, they did not think MSM would prefer RM suppositories or enemas. 
Cost was a major concern. Some participants thought that as sex workers have a large number of partners per day, paying for one or more tubes of RM per day would be costly. For instance, a double-decker said,

Usually after giving 'gaans' [receptive anal sex] they will go to toilet, wash and then go to other person. It seems like a total tube will be used in a day. One needs to spend more money for buying it then. (Double-decker)

In fact, kothis and others who engage in sex work wanted to receive free or subsidized RMs from CBOs, similar to the free condoms they receive from these organizations: In medical shops it [RM] won't be provided free of cost. It can be distributed through $\mathrm{CBOs}$ for kothis, either free or in decreased price. Better if subsidized because if provided free, both educated and illiterate kothis may think it may not be effective. So, it can be distributed through CBOs at a subsidized price of rupees 10 per sachet. (Kothi in sex work)

Some MSM felt that options should be available for accessing RMs in government hospitals and pharmacies. However, other participants reported that they would hesitate to buy RMs from pharmacies unless marketed as a general sexual lubricant. For instance, a bisexual man said,

When we get condoms from medical shops they may think that we have sex with females, but if we ask for rectal microbicide they may think, "He is going to have sex with a guy." (Bisexual man)

A few reported that they would prefer going to pharmacies that are away from home if they need to buy RMs, to preserve their privacy.

Finally, participants believed that RM uptake among MSM will depend on its effectiveness; if it provided $90 \%$ protection against HIV infection, participants felt it would result in far greater usage than if it were only $50 \%$ effective.

\section{Social and Community Contexts}

Subjective and community norms. MSM expressed concerns about the stigma of being judged to be sexually promiscuous or suspected to be a sex worker if one uses RMs. Double-deckers and kothis who are flexible in their sexual roles were concerned that having a rectal use product might suggest that the person only takes a receptive role. Thus, buying and carrying RMs could be a stigmatizing activity. Contrarily, kothis who openly engage in sex work reported that 
carrying condoms and RMs would indicate to their clients that they are responsible and safe, and thus they could earn more. Also, kothis in sex work have their own friendship networks that are unlikely to judge them if they use RMs, as condom use and lubricant use are accepted norms within these networks.

Facilitating and hindering conditions. Given that condom use seems to have been established as a safer sex norm among MSM communities, use of RMs could be socially marketed as denoting one's personal responsibility to take care of one's own and one's partner's health. The presence of strong community infrastructure in the form of $\mathrm{CBO}$ in different parts of India provides opportunities to disseminate information about RMs and distribute RM products to atrisk MSM. Having sex in cruising areas, however, was described as a hindering condition to RM use. Participants noted that RM use would be feasible only if sex happens in private places but not in cruising sites where there will not be time or privacy to apply a RM. As a peer counselor explained in regard to sex workers,

It [rectal microbicide] will be useful only for those MSM in sex work who have sex in a lodge because each time after sex he can go to the toilet, wash and re-apply it before calling the next client. Consider if someone has sex in a bush or railway track. It is not at all possible. (MSM peer counselor)

Similarly, other MSM may travel directly from their workplaces to cruising sites and thereby not have a chance to apply a RM beforehand.

Alcohol use was identified as another hindering condition to RM use. A community leader was pessimistic in that even if RMs are provided for free, one cannot be sure whether people will use it, including an applicator, properly:

If they drink alcohol may either forget or don't consider using [a rectal microbicide].

Another problem is with the applicator. So how will we ensure that people will use it properly? (MSM community leader)

\section{Discussion}

In this study of RM acceptability among diverse MSM in India, we identified low awareness but strong openness to use a future RM. Our findings further reveal technological, individual, and community contexts that may influence RM acceptability and preferences.

In accordance with the Technology Acceptance Model, the technological context of RM acceptability revealed gel was the preferred formulation, consistent with participants' current use 
of water-based lubricants. MSM in Thailand similarly identified preferences for gel formulation over suppository, in addition to concerns about the ease of RM product use (Newman et al., 2016; Newman et al., 2013). MSM in the United States indicated higher acceptability for a lubricant-filled applicator than a suppository or enema (Pines et al., 2013). Suppositories and enemas were similarly not preferred delivery systems in our study, but a U.S. study reported MSM preferred enema-administered RM gel over suppositories (Carballo-Diéguez et al., 2008). Specific concerns emerged in the present study about difficulties in using a RM applicator, similar to MSM and transgender women, including male and transgender female sex workers, in the United States and Puerto Rico (Giguere, Dolezal, et al., 2016; Giguere, Frasca, et al., 2016). Our findings suggest that multiple delivery options for RMs may be ideal for MSM in India and that future trials should systematically evaluate preferences in relation to both formulations and delivery systems (lubricant/gel, enema or suppository, applicator type), depending on available candidate RMs.

Although in the technological context gel was the preferred RM formulation, this preference was further influenced by individual contexts such as engagement in sex work and number of sexual partners. A RM gel that is colorless and non-leaky, and which is easy to apply and requires less frequent application for those with multiple sex partners, might be more acceptable across diverse MSM subgroups in India. The future implementation of RMs needs to consider daily life contexts of MSM, particularly those who may be most vulnerable, in terms of the geographical locations in which RMs would be used (cruising site or private place), how frequently they might need to be applied in a given day (single or multiple encounters) and how far in advance before potential sexual encounters they could be applied and still be effective. The specific ramifications of the interaction between technological and individual contexts will need to be addressed once RMs are licensed for use and the parameters around duration of protection and frequency of recommended application are indicated; herein, clinical trials that integrate the assessment of social-behavioral with biomedical factors may help to inform prevention strategies that anticipate and address the interplay between technological and individual contexts in product roll-out (Newman, Duan, Kakinami, \& Roberts, 2008; Newman et al., 2013).

Individual and interpersonal contexts of the Technology Acceptance Model revealed that MSM in sex work, predominantly kothis, who are open about their sexual identity and sex work, preferred using RMs; they regarded a RM as an additional tool for HIV prevention, especially as it could be used in certain contexts of condomless sex - for example, with male steady partners or in all too frequent instances of forced sex (Newman, Chakrapani, et al., 2008). In addition, 
MSM in sex work perceived that lubricant-like RMs would have multiple advantages—rapid turnover of satisfied clients (due to decreased sex duration) and possibility of earning more money by marketing oneself as safe and responsible. These perceived advantages of RMs were similarly indicated among male sex workers in Thailand who were more accepting of RM products with less than ideal attributes (i.e., partial efficacy) than other MSM, though with additional concerns about the cost of products, particularly if they required multiple applications (Newman et al., 2016). Identifying population-specific RM preferences and needs among male and transgender female sex workers is especially important given the vulnerable contexts in which they work and their high risk for HIV infection (Baral et al., 2015; Newman et al., 2016). Interactions between personal and community contexts were observed through the influence of $\mathrm{CBO}$ in fostering safer sex practices among MSM, including those who engage in sex work. As MSM in many Indian cities currently receive free condoms and water-based lubricants from CBOs (Saggurti et al., 2013; Singh, Dasgupta, Patankar, \& Sinha, 2013), they similarly expected CBOs to provide free or subsidized RMs. MSM were willing to use RMs if recommended by CBOs. The trust MSM reported in CBOs suggests that conducting RM trials in collaboration with $\mathrm{CBO}$ s serving MSM communities may support recruitment and follow-up, with more efficient trial recruitment than through hospital-based settings.

Condom use is often inconsistent within steady relationships and not a norm among some MSM in India (Chakrapani, Boyce, Newman, \& Row Kavi, 2013; Ramanathan et al., 2013). In such interpersonal contexts, we found that potential stealth use of RMs poses opportunities (protection of self or partner against HIV) as well as risks (relationship conflict if partner finds out) for MSM with steady male partners. Similarly, studies in Thailand and the United States reported trust in steady partners as both a facilitator and a barrier to RM use (Giguere, Dolezal, et al., 2016; Newman et al., 2013). In accordance with the Technology Acceptance Model, this suggests the importance of individual and organizational contexts in RM acceptability, and that depending on the context clandestine use may be a barrier or a facilitator for HIV prevention. From an individual context, future roll-out of RMs should include information and skills training for MSM to safely navigate the potential risks and benefits of covert use. From an organizational context, future roll-out of RMs may provide renewed opportunities for interventions to enhance sexual communication and safer sex negotiation skills among MSM, thereby supporting positive community norms about RM use and combination HIV prevention. Use of RMs, and safer sex products in general, might best be promoted as a shared responsibility in the case of steady relationships-as panthis in this study perceived that use of RMs was the responsibility of their kothi partners. 
Finally, we found that social and community contexts influence RM acceptability. Divergent perspectives on the provision of free or subsidized RMs, and CBO, government, or private pharmacy venues for distribution, for example, appeared to reflect socioeconomic differences and concerns about stigma. For some higher socioeconomic status MSM, the cost of RMs may be incidental, and distribution through $\mathrm{CBO}$ s may present barriers due to concerns about disclosing their sexuality, mixing with "lower class" MSM, and anticipated stigma due to being presumed to be at risk for HIV infection (Chakrapani et al., 2007; Shahani, 2008). These middle- and upper-middle class MSM may prefer to procure RMs from conveniently located pharmacies, but features of labeling and packaging of a RM that minimize stigma and embarrassment may support their comfort in the pharmacy setting. For lower socioeconomic status MSM, including kothis and others engaged in sex work, purchasing a RM at full price in a pharmacy would likely be prohibitive, and antithetical to existing norms of CBO-provided condoms and lubricant (NACO, 2016); nevertheless a few participants expressed suspicions regarding the quality of a new product provided for free. This diversity of perspectives argues against a one-size-fits-all approach to distribution and marketing of RMs; rather, product availability through different types of venues and pricing levels, in the context of CBO and health provider assurances of product safety, may facilitate uptake across diverse MSM communities.

Further differences across subgroups of MSM also reflect the influence of social and community contexts on RM acceptability, and the importance of engaging diverse groups of MSM in RM research. Panthis (masculine and insertive role) and gay-identified men in this study perceived that RMs may be more relevant to kothis (who primarily engage in receptive anal sex) and MSM who have multiple sex partners-especially MSM in sex work. In turn, although RMs are health promoting, they were perceived as conveying stigma. In addition to providing accurate information about the advantages of future RMs, interventions within MSM communities to address multifaceted stigma associated with the receptive sexual role, with sex work, and with HIV, may support RM uptake. In Thailand, higher levels of HIV stigma were associated with lower acceptability of a future RM among young MSM and transgender women, including male and transgender female sex workers (Logie, Newman, Weaver, Roungprakhon, \& Tepjan, 2016).

The informal social networks of MSM created as a byproduct of community mobilization efforts to support HIV preventive interventions seem to have promoted positive social norms on condom use (Saggurti et al., 2013), especially among kothis, many of whom engage in sex work. Thus, as reflected in the social and community contexts domain of our adapted Technology Acceptance Model, promotion of positive community norms on RM use may broadly 
facilitate uptake of future RMs, including among MSM in sex work. Among double-deckers and gay men, challenges emerged from the concern that bringing up RM use to their sexual partners would be construed as suggesting that their partners adopt the receptive role in anal sex. This reinforces the importance of promoting positive community norms about safer sex, including use of RMs, as well as enhancing sexual communication skills among diverse subgroups of MSM to support HIV prevention.

\section{Limitations and Strengths}

The inclusion of diverse MSM subgroups in two Indian cities revealed in-depth perspectives and contexts of acceptability of future RMs. As participants were recruited primarily through $\mathrm{CBOs}$, some of which conduct targeted outreach for sex workers, their perspectives might differ from MSM who are not CBO-affiliated. The higher education, income and greater proportion of gayidentified men in the Mumbai sample, and the higher proportion of sex workers in the Chennai sample reflect the $\mathrm{CBO}$ clienteles in the different cities. Chennai lacks $\mathrm{CBO}$ s that include services tailored for gay-identified men in contrast to other MSM subgroups, with CBOs predominantly focusing on MSM engaged in sex work, a population at elevated risk for HIV infection (Baral et al., 2015; Newman, Chakrapani, et al., 2008). Nevertheless, selective differences in RM acceptability associated with particular MSM subgroups appeared to apply across the two cities. Overall, while our findings may be transferrable to other urban settings in India with targeted HIV interventions among MSM, as in any qualitative investigation, the generalizability of the findings needs to be examined in further research among representative samples. The results provide a rich basis from which to conduct further RM acceptability research and clinical trials in India. Finally, by necessity, we explored acceptability of hypothetical RMs; once RMs are licensed, and specific product attributes (e.g., duration of protection) are known, product acceptability and concerns may change. Our innovative use of pictorial representations of hypothetical RM products helped to provide clear descriptions, which facilitated participants' expressions of the nuanced factors described that influence acceptability of this new technology under development.

\section{Conclusion}

Using a modified Technology Acceptance Model, this study highlights a range of factors and focal contexts that may facilitate the introduction of RMs once they are available. The technological context of RM introduction, including use of condoms and water-based lubricants, as well as individual and interpersonal contexts, may influence the acceptability and 
effectiveness of future RMs - as well as participation in RM clinical trials. In addition, our modifications to the Technology Acceptance Model that situate technological contexts within social and community domains enable a more comprehensive understanding of possible barriers; these include stigmatization of a rectal use product within MSM communities and logistical challenges in using RMs in cruising areas and sex work sites. These findings may support the design, testing and dissemination of user-friendly RM products, and promotion of RM use among diverse subgroups of Indian MSM. India, with a concentrated HIV epidemic among MSM and transgender women, needs to build its evidence base to support the design and implementation of RM trials in the near future to increase HIV prevention options among these key populations at risk.

\section{Acknowledgments}

The authors acknowledge Vivek R. Anand and Ashok Row Kavi, The Humsafar Trust; Sunil Menon and Jaya, Sahodaran; and Sekar Balasubramaniam, Social Welfare Association for Men (SWAM), for their help and leadership in successful implementation of the study. The authors thank all the study participants for sharing their perspectives.

\section{Declaration of Conflicting Interests}

The authors declared no potential conflicts of interest with respect to the research, authorship, and/or publication of this article.

\section{Funding}

The authors disclosed receipt of the following financial support for the research, authorship, and/or publication of this article: This research was supported by grants from the Canadian Institutes of Health Research (MOP-102512; THA-118570, principal investigator [PI]: Newman). Peter A. Newman is supported by a Canada Research Chair in Health and Social Justice. 


\section{References}

Aggelidis, V. P., \& Chatzoglou, P. D. (2009). Using a modified technology acceptance model in hospitals. International Journal of Medical Informatics, 78, 115-126.

Anton, P. A., Saunders, T., Elliott, J., Khanukhova, E., Dennis, R., Adler, A., . . McGowan, I. (2011). First phase 1 double-blind, placebo-controlled, randomized rectal microbicide trial using UC781 gel with a novel index of ex vivo efficacy. PLoS ONE, 6(9), e23243.

Baral, S. D., Friedman, M. R., Geibel, S., Rebe, K., Bozhinov, B., Diouf, D., . . Cáceres, C. F. (2015). Male sex workers: Practices, contexts, and vulnerabilities for HIV acquisition and transmission. The Lancet, 385, 260-273.

Baral, S. D., Sifakis, F., Cleghorn, F., \& Beyrer, C. (2007). Elevated risk for HIV infection among men who have sex with men in lowand middle-income countries 2000-2006: A systematic review. PLoS Medicine, 4(12), e339.

Brink, P. J., \& Wood, M. J. (1998). Advanced design in nursing research (2nd ed.). Thousand Oaks, CA: Sage.

Carballo-Diéguez, A., Dolezal, C., Bauermeister, J. A., O’Brien, W., Ventuneac, A., \& Mayer, K. (2008). Preference for gel over suppository as delivery vehicle for a rectal microbicide: Results of a randomised, crossover acceptability trial among men who have sex with men. Sexually Transmitted Infections, 84, 483-487.

Chakrapani, V., Boyce, P., Newman, P. A., \& Row Kavi, A. (2013). Contextual influences on condom use among men who have sex with men in India: Subjectivities, practices and risks. Culture, Health \& Sexuality, 15, 938-951.

Chakrapani, V., Newman, P.A., Shunmugam, M., McLuckie, A., \& Melwin, F. (2007). Structural violence against kothi-identified men who have sex with men in Chennai, India: A qualitative investigation. AIDS Education and Prevention, 19, 346-364.

Chakrapani, V., Shunmugam, M., Newman, P. A., Kershaw, T., \& Dubrow, R. (2015). HIV status disclosure and condom use among HIV-positive men who have sex with men and hijras (male-to-female transgender people) in India: Implications for prevention. Journal of HIVIAIDS \& Social Services, 14, 26-44.

Cranston, R., Lama, J. R., Richardson, B. A., Carballo-Diéguez, A., Kunjara Na Ayudhya, R., Liu, K., . . McGowan, I. (2017). MTN-017: A rectal phase 2 extended safety and acceptability study of tenofovir reduced-glycerin $1 \%$ gel. Clinical Infectious Diseases, 64 , 614-620.

Creswell, J. W., \& Miller, D. L. (2000). Determining validity in qualitative inquiry. Theory Into Practice, 39, 124-131. 
Davis, F. D. (1989). Perceived usefulness, perceived ease of use, and user acceptance of information technology. MIS Quarterly, 13, 319-340.

Denzin, N. K. (1978). Sociological methods: A sourcebook. New York: McGraw-Hill.

Fusch, P. I., \& Ness, L. R. (2015). Are we there yet? Data saturation in qualitative research. Qualitative Report, 20, 1408-1416.

Gagnon, M. P., Orruno, E., Asua, J., Abdeljelil, A. B., \& Emparanza, J. (2012). Using a modified technology acceptance model to evaluate healthcare professionals' adoption of a new telemonitoring system. Telemedicine Journal and E-Health, 18, 54-59.

Giguere, R., Dolezal, C., Bauermeister, J. A., Frasca, T., Valladares, J., Febo, 1., . . CarballoDieguez, A. (2016). Influence of partner type on acceptability and likelihood of use of a rectal microbicide among young men who have sex with men in the United States and Puerto Rico. Journal of Sex Research, 53, 633-641.

Giguere, R., Frasca, T., Dolezal, C., Febo, I., Cranston, R. D., Mayer, K., . . Carballo-Diéguez, A. (2016). Acceptability of three novel HIV prevention methods among young male and transgender female sex workers in Puerto Rico. AIDS and Behavior, 20, 2192-2202.

Greene, E., Batona, G., Hallad, J., Johnson, S., Neema, S., \& Tolley, E. E. (2010). Acceptability and adherence of a candidate microbicide gel among high-risk women in Africa and India. Culture, Health \& Sexuality, 12, 739-754.

Hiruy, H., Fuchs, E. J., Marzinke, M. A., Bakshi, R. P., Breakey, J. C., Aung, W. S., . . Hendrix, C. W. (2015). A phase 1 randomized, blinded comparison of the pharmacokinetics and colonic distribution of three candidate rectal microbicide formulations of tenofovir $1 \%$ gel with simulated unprotected sex (CHARM-02). AIDS Research and Human Retroviruses, 31, 1098-1108.

International Rectal Microbicide Advocates. (2010). Rectal microbocides 101. Retrieved from http://www.global-campaign.org/clientfiles/IRMA_RM_101_FS_2010.pdf

Jha, U. M., Raj, Y., Venkatesh, S., Dhingra, N., Paranjpe, R. S., \& Saggurti, N. (2014). HIV epidemic among men who have sex with men in India: National scenario of an unfinished agenda. HIVIAIDS, 6, 159-170.

Joglekar, N. S., Joshi, S. N., Deshpande, S. S., Parkhe, A. N., Katti, U. R., \& Mehendale, S. M. (2010). Acceptability and adherence: Findings from a Phase II study of a candidate vaginal microbicide, "praneem polyherbal tablet," in Pune, India. Transactions of the Royal Society of Tropical Medicine \& Hygiene, 104, 412-415.

Karim, Q. A., Karim, S. S. A., Frohlich, J. A., Grobler, A. C., Baxter, C., Mansoor, L. E., . . CAPRISA 004 Trial Group. (2010). Effectiveness and safety of tenofovir gel, an 
antiretroviral microbicide, for the prevention of HIV infection in women. Science, 329, 11681174.

Lincoln, Y. S., \& Guba, E. G. (1985). Naturalistic inquiry. Newbury Park, CA: Sage.

Logie, C., Newman, P. A., Weaver, J., Roungprakhon, S., \& Tepjan, S. (2016). HIV-related stigma and HIV prevention uptake among young men who have sex with men and transgender women in Thailand. AIDS Patient Care and STDs, 30, 92-100.

McGowan, I. (2011). Rectal microbicides: Can we make them and will people use them? AIDS and Behavior, 15(Suppl. 1), S66-S71.

McGowan, I., Hoesley, C., Cranston, R. D., Andrew, P., Janocko, L., Dai, J. Y., . . Mayer, K. (2013). A phase 1 randomized, double blind, placebo controlled rectal safety and acceptability study of tenofovir 1\% gel (MTN-007). PLoS ONE, 8(4), e60147.

Microbicide Trials Network. (2013). Microbicides: A promising strategy. Retrieved from www.mtnstopshiv.org/node/82

National AIDS Control Organization. (2016). Annual report 2015-16. Retrieved from http://164.100.12.21/ upload/2016\%20Data/Annual\%20Report/Annual\%20 Report\%202015-16_NACO.pdf

Newman, P. A., Cameron, M. P., Roungprakhon, S., Tepjan, S., \& Scarpa, R. (2016). Acceptability and preferences for hypothetical rectal microbicides among a community sample of young men who have sex with men and transgender women in Thailand: $A$ discrete choice experiment. AIDS and Behavior, 20, 2588-2601.

Newman, P. A., Chakrapani, V., Cook, C., \& Kakinami, L. (2008). Correlates of paid sex among men who have sex with men in Chennai, India. Sexually Transmitted Infections, 84, 434438.

Newman, P. A., Duan, N., Kakinami, L., \& Roberts, K. (2008). What can HIV vaccine trials teach us about dissemination? Vaccine, 26, 2528-2536.

Newman, P. A., Roungprakhon, S., \& Tepjan, S. (2013). A social ecology of rectal microbicide acceptability among young men who have sex with men and transgender women in Thailand. Journal of the International AIDS Society, 16, Article 18476.

Newman, P. A., Roungprakhon, S., Tepjan, S., \& Yim, S. (2010). Preventive HIV vaccine acceptability and behavioral risk compensation among high-risk men who have sex with men and transgenders in Thailand. Vaccine, 28, 958-964.

Orruno, E., Gagnon, M. P., Asua, J., \& Abdeljelil, A. B. (2011). Evaluation of teledermatology adoption by health-care professionals using a modified Technology Acceptance Model. Journal of Telemedicine and Telecare, 17, 303-307. 
Patton, M. Q. (1999). Enhancing the quality and credibility of qualitative analysis. Health Sciences Research, 34(5, Pt. 2), 1189-1208.

Pines, H. A., Gorbach, P. M., Weiss, R. E., Hess, K., Murphy, R, Saunders, T., . . Cranston, R. D. (2013). Acceptability of potential rectal microbicide delivery systems for HIV prevention: A randomized crossover trial. AIDS and Behavior, 17, 1002-1015.

Pope, C., Ziebland, S., \& Mays, N. (2000). Qualitative research in health care: Analysing qualitative data. BMJ, 320, 114-116.

Ramanathan, S., Chakrapani, V., Ramakrishnan, L., Goswami, P., Yadav, D, Subramanian, T., . .. Paranjape, R. (2013). Consistent condom use with regular, paying, and casual male partners and associated factors among men who have sex with men in Tamil Nadu, India: Findings from an assessment of a large-scale HIV prevention program. BMC Public Health, 13, Article 827.

Ritchie, J., \& Lewis, J. (2003). Qualitative research practice: A guide for social science students and researchers. Thousand Oaks, CA: Sage.

Saggurti, N., Mishra, R. M., Proddutoor, L., Tucker, S., Kovvali, D., Parimi, P., \& Wheeler, T. (2013). Community collectivization and its association with consistent condom use and STI treatment-seeking behaviors among female sex workers and high-risk men who have sex with men/transgenders in Andhra Pradesh, India. AIDS Care, 25(Suppl. 1), S55-S66.

Shahani, P. (2008). Gay Bombay: Globalization, love and (be) longing in contemporary India. New Delhi, India: Sage.

Singh, S., Dasgupta, S., Patankar, P., \& Sinha, M. (2013). A people stronger: The collectivization of MSM and TG groups in India. New Delhi, India: Sage.

Solomon, S. S., Mehta, S. H., Srikrishnan, A. K., Vasudevan, C. K., McFall, A. M., Balakrishnan, P., . . Celentano, D. D. (2015). High HIV prevalence and incidence among MSM across 12 cities in India. AIDS, 29, 723-731.

Stein, Z. A. (1990). HIV prevention: The need for methods women can use. American Journal of Public Health, 80, 460-462.

Tang, E. C., Galea, J. T., Kinsler, J. J., Gonzales, P., Sobieszczyk, M. E., Sanchez, J., \& Lama, J. R. (2016). Using conjoint analysis to determine the impact of product and user characteristics on acceptability of rectal microbicides for HIV prevention among Peruvian men who have sex with men. Sexually Transmitted Infections, 92, 200-205.

Tolley, E. E., Eng, E., Kohli, R., Bentley, M. E., Mehendale, S., Bunce, A., \& Severy, L. J. (2006). Examining the context of microbicide acceptability among married women and men in India. Culture, Health \& Sexuality, 8, 351-369. 
Vittinghoff, E., Douglas, J., Judson, F., McKirnan, D., MacQueen, K., \& Buchbinder, S. P. (1999). Per-contact risk of human immunodeficiency virus transmission between male sexual partners. American Journal of Epidemiology, 150, 306-311. 